\title{
A PROOF OF THE CORONA CONJECTURE FOR FINITE OPEN RIEMANN SURFACES ${ }^{1}$
}

\author{
BY NORMAN L. ALLING \\ Communicated by A. M. Gleason, September 17, 1963
}

For an open Riemann surface $X$ the corona conjecture is the following: let $B(X)$ be the algebra of bounded analytic functions on $X$ and let $\mathfrak{M}(X)$ be the space of maximal ideals of $B(X)$; then $X$ is dense in $\mathfrak{M}(X)$. Carleson [3] has proved that the corona conjecture is true for the open unit disk $D$. We will sketch a proof of the following extension of Carleson's Theorem.

THEOREM. If $X$ is a finite open Riemann surface, then $X$ is dense in $\mathfrak{M}(X)$.

By a finite open Riemann surface is meant a proper, open, connected subset of a compact Riemann surface $W$ whose boundary $\Gamma$ is also the boundary of $W-X$ and consists of a finite number of closed analytic arcs. Since $W-X$ has an interior we may employ the Riemann-Roch Theorem to show that $B(X)$ has enough functions to separate points and provide each point in $X$ with a local uniformizer. Such a surface $X$ therefore admits a natural homeomorphic imbedding into $\mathfrak{M}(X)$; thus the corona conjecture is seen to be meaningful.

Let $X$ be a finite open Riemann surface. Ahlfors [1] has shown that there exists an analytic mapping $p_{0}$ of $\bar{X}$ into the plane such that $p=p_{0} \mid \bar{X}$ is an $n$-fold covering of $X$ onto $D$ and $p_{0}(\Gamma)=\bar{D}-D$. Since $\Gamma$ consists of closed analytic arcs, no ramification occurs on $\bar{D}-D$. Clearly $p^{*}$, the adjoint of $p$, is a $C$-isomorphism of $B(D)$ into $B(X)$, $C$ being the complex field. Let $B(D)^{*}$ denote the range of $p^{*}$, and for $f \in B(D)$ let $p^{*}(f)=f^{*}$.

Let $\sigma_{k}$ denote the $k$ th elementary symmetric function on $n$ letters. For $z \in D$ let $p^{-1}(z)=\left\{x_{1}(z), \cdots, x_{n}(z)\right\}$, each appearing to its multiplicity. Given $f \in B(X), \sigma_{k}\left(f\left(x_{1}(z)\right), \cdots, f\left(x_{n}(z)\right)\right)$ is in $B(D)$. Thus, as is well known, $B(X)$ is integrally dependent on $B(D)^{*}$.

Given $N \in \mathfrak{M}(X)$ let $M^{*}=N \cap B(D)^{*}$ and let $P(N)=\left(p^{*}\right)^{-1}\left(M^{*}\right)$. Since $\mathfrak{M}(X)$ and $\mathfrak{M}(D)$ have the weak topology, $P$ is continuous. Further, $P$ is an extension of $p$. Since $B(X)$ is integrally dependent on $B(D)^{*}, P$ is surjective. For $f \in B(D)((B(X))$ let $\hat{f}$ denote the natural extension of $f$ to $\mathfrak{M}(D)((\mathfrak{M}(X))$. (See Hoffman [4, Chapter 10] for details.) Given $f \in B(D), \hat{f} P=f^{*}$. Let $z$ denote the identity function

1 This research was supported in part by National Science Foundation grant NSF. GP-379. 
on $D$ and let $\pi=z$. Clearly $\pi$ is a continuous mapping of $\mathfrak{M}(D)$ onto $\bar{D}$, which is the identity on $D$. We need an analogous function on $\mathfrak{M}(X)$.

Let $B_{C}(X)=\{f \in B(X): f$ has a continuous extension on $\bar{X}\}$. Arens [2] has shown that the maximal ideal space of $B_{C}(X)$ is naturally homeomorphic to $\bar{X}$, with which we will identify it. For $N \in \mathfrak{M}(X)$ let $\Pi(N)=N \cap B_{C}(X)$. $\Pi$ is a continuous mapping of $\mathfrak{M}(X)$ onto $\bar{X}$, which is the identity on $X$. One can show that $p_{0} \Pi=\pi P$; thus given $N \in \mathfrak{M}(X)$ such that $P(N) \in D, N$ is in $X$. Let $\gamma \in \Gamma, \alpha=p_{0}(\gamma)$, $\mathfrak{M}(X)_{\gamma}=\Pi^{-1}(\gamma)$, and let $\mathfrak{M}(D)_{\alpha}=\pi^{-1}(\alpha)$. Clearly $P\left(\mathfrak{M}(X)_{\gamma}\right) \subset \mathfrak{M}(D)_{\alpha}$.

Let $K(X), K(D)^{*}, K_{C}(X)$, and $K_{C}(D)^{*}$ be the quotient fields of $B(X), B(D)^{*}, B_{C}(X)$, and $B_{C}(D)^{*}$, respectively, in the field of all meromorphic functions on $X$. The elementary symmetric functions can be used, as they were above, to define mappings from $K(X)$ into $K(D)^{*}$ and from $K_{C}(X)$ into $K_{C}(D)^{*}$, with the aid of which it can be shown that $K(X) / K(D)^{*}$ and $K_{C}(X) / K_{C}(D)^{*}$ are algebraic extensions of degree $n$. The coefficients of the field polynomial of the extension and the respective symmetric functions are the same. Given $g \in B_{C}(X)$ and $\alpha \in \bar{D}-D$ such that the continuous extension $\bar{g}$ of $g$ to $\bar{X}$ assumes $n$ distinct values on $p_{0}^{-1}(\alpha)$, then $g$ generates $K(X), K_{C}(X)$ over $K(D)^{*}, K_{C}(D)^{*}$ respectively. The discriminate $d^{*}$ of $g$ is in $B_{C}(D)^{*}$. Its extension $\bar{d}$ to $\bar{D}$ is nonzero at $\alpha$, since $g$ assumes distinct values on $p_{0}^{-1}(\alpha)$. Since $g$ generates $K(X)$ over $K(D)^{*}$ and $K_{C}(X)$ over $K_{C}(D)^{*}$, given $b \in B(X)\left(\left(B_{C}(X)\right)\right.$ there exist unique $f_{0}, \cdots, f_{n-1} \in K(D)\left(K_{C}(D)\right)$ such that $b=\sum_{j=0}^{n-1} f_{j}^{*} g^{j}$. Using Cramer's rule, one can find unique $a_{0}, \cdots, a_{n-1} \in B(D)\left(B_{C}(D)\right)$ such that $f_{j}=a_{j} / d$. With the aid of this elementary field theory the following can be proved: $P \mid \mathfrak{M}(X)_{\gamma}$ is an injection onto $\mathfrak{M}(D)_{\alpha}$.

By the nature of $p_{0}$, we can choose a closed neighborhood $V$ of $\alpha$ in $\bar{D}$ such that if $U$ is the component of $p_{0}^{-1}(V) \cap \bar{X}$ that contains $\gamma$, then $p_{0} \mid U$ is a homeomorphism. Let $v=\pi^{-1}(V)$ and let $\mathcal{U}=\Pi^{-1}(U)$. Since $\mathfrak{M}(X)$ and $\mathfrak{M}(D)$ are compact, $\mathcal{u}$ and $\mathcal{U}$ are compact. Since $P \mid \mathfrak{M}(X)_{\gamma}$ is an injective mapping onto $\mathfrak{M}(D)_{\alpha}$, and by the choice of $U$ and $V, P \mid \mathcal{u}$ is an injection onto $\vartheta$. Since $\mathfrak{u}$ is compact $P \mid \mathcal{u}$ is a homeomorphism. From this it follows easily that $P$ is an open mapping. Invoking Carleson's Theorem [3], for the first time, we find that $X$ is dense in $\mathfrak{M}(X)$, proving the theorem.

Actually, we have proved somewhat more. Let $\Gamma_{1}, \cdots, \Gamma_{k}$ be the components of $\Gamma$. By definition, these components are nondegenerate, closed, analytic curves in $W$ that $p_{0}$ takes to $S$, the unit circle. Since $\mathfrak{M}(D)_{\alpha}$ is connected for each $\alpha \in S$ [4], $\mathfrak{M}(X)_{\gamma}$ is connected for each $\gamma \in \Gamma$; thus $\mathfrak{M}(X)_{\Gamma_{i}}\left(=\Pi^{-1}\left(\Gamma_{i}\right)\right)$ is connected for each $i$. We conclude 
that $\mathfrak{M}(X)_{\Gamma_{1}}, \cdots, \mathfrak{M}(X)_{\Gamma_{k}}$ are the components of $\mathfrak{M}(X)-X$. Let $q_{i}$ be the number of times $\Gamma_{i}$ covers $S$ under $p_{0}$; clearly $\sum_{i=1}^{k} q_{i}$ equals $n$. $P \mid \mathfrak{M}(X)_{\Gamma_{i}}$ is a local homeomorphism of $\mathfrak{M}(X)_{\Gamma_{i}}$ onto $\mathfrak{M}(D)-D$, that covers each point $q_{i}$ times.

Let $Y$ be an open Riemann surface and suppose there exists a conformal homeomorphism of $Y$ onto a finite open Riemann surface $X$. Then clearly we can carry the solution of the corona conjecture to $Y$.

REMARK. Using recent results of Röhrl [5, Theorem 4.2], one can show that $B(X)$ is a free $B(D)^{*}$-module of dimension $n$, a basis for which can be chosen in $B_{C}(X)$. With this one can show that $P \mid \mathfrak{M}(X)_{\gamma}$ is injective. It seems, at this time, not unreasonable to conjecture that an element $g \in B_{C}(X)$ can be found such that $1, g, \cdots, g^{n-1}$ is a free basis of $B(X)$ over $B(D)^{*}$. From this it would follow immediately that $P\left(\mathfrak{M}(X)_{\gamma}\right)=\mathfrak{M}(D)_{\alpha}$ (this can also be shown using Carleson's Theorem).

Acknowledgments. I am indebted to Professors Gleason, Hoffman, Röhrl, and Royden, with whom I had several very useful discussions during the course of this research.

\section{REFERENCES}

1. L. Ahlfors, Open Riemann surfaces and extremal problems on compact subregions, Comment. Math. Helv. 24 (1950), 100-134.

2. R. Arens, The closed maximal ideals of algebras of functions holomorphic on a Riemann surface, Rend. Circ. Mat. Palermo (2) 7 (1958), 1-13.

3. L. Carleson, Interpolations by bounded analytic functions and the corona problem, Ann. of Math. (2) 76 (1962), 547-559.

4. K. Hoffman, Banach spaces of analytic functions, Series in modern analysis, Prentice-Hall, Engelwood Cliffs, N. J., 1962.

5. H. Röhrl, Unbounded coverings of Riemann surfaces and extensions of rings of meromorphic functions, Trans. Amer. Math. Soc. 107 (1963), 320-346.

MASSACHUSETTS INSTITUTE OF TEChNOLOGY AND PURdue University 